Ann. Abeille, 1964, 7 (I), 23-45.

\title{
LES RÉGULATIONS THERMIQUES DANS LA RUCHE EN ÉTÉ
}

\author{
Y. LENSKY \\ Université hébraïque de Jérusalem, \\ Faculté d'Agriculture, Rehovot (Isrä̈l)
}

SOMMAIRE

L'auteur a étudié au moyen de thermocouples le microclimat de la ruche exposée aux conditions subtropicales de l'été israélien. Il tire de ce travail des conclusions quant à la possibilité de protéger efficacement les ruches contre le soleil par un revêtement convenable. Il met en évidence les conséquences fâcheuses pour la récolte de miel d'un effort exagéré de thermorégulation demandé aux abeilles. Il expose un procédé d'approvisionnement continu en eau des ruches qui assure une augmentation de la ponte de la reine.

\section{INTRODUC'TION}

Le nid des Insectes sociaux présente, d'une façon très générale, des phénomènes de régulation des facteurs climatologiques qui sont rendus possibles par la division du travail au sein de la colonie. Les conditions optimales de température et d'hygrométrie qui règnent dans le nid permettent un développement larvaire plus rapide que chez les Insectes solitaires, lesquels sont directement soumis à 1'influence du milieu ambiant. On peut donc considérer qu'il $\mathrm{y}$ a, de ce point de vue, supétiorité des insectes sociaux (ALLEE et al, I950).

Chez l'Abeille (Apis mellifica, L) la température du nid à couvain est relativement très stable et indépendante de la température extérieure (RIBBANDs, I953). Il existe donc une régulation thermique, laquelle n'a guère été étudiée, aussi bien en Europe qu'en Amérique, que dans le sens d'une lutte contre les basses températures extérieures ; les réactions à l'égard d'une chaleur excessive sont moins connues Or, les problèmes posés par la régulation thermique chez les Abeilles en pays froid ou tempéré sont tout à fait différents de ceux qui se posent dans les pays subtropicaux. Dans les premiers, l'activité des ouvrières et la ponte de la reine cessent souvent complètement pendant l'hiver. Dans les seconds, il n'y a pas d'hivernage 
(BodenheImer et al., I937) et le problème essentiel pour les abeilles est celui de la climatisation du nid pendant les fortes chaleurs de l'été.

C'est pourquoi, dans la présente étude, nous avons étudié, dans des conditions naturelles, le comportement des abeilles au cours de l'été israélien. Nous avons examiné l'influence du régime thermique sur la thermorégulation et sur la récolte du miel. Nous avons également étudié l'action d'un apport artificiel d'eau (sous forme d'aspersion) sur la ponte de la reine. Nous avons tenu compte pour réaliser ce travail des remarques de Wellingron (I957) qui insiste sur l'importance des facteurs du milieu dans les études sur le comportement des Insectes.

Le travail que nous présentons vient en complément de deux études précédentes. La première (LENSky, I964) concerne la capacité des colonies d'abeilles de stabiliser la température du nid à couvain pendant les très fortes chaleurs. La seconde (LENSKY, I958) se rapporte au microclimat de la ruche vide. Rappelons brièrement que nous avons montré que le maximum de température enregistré à l'intérieur de ruches non peuplées varie en fonction du revêtement et en fonction de la nature de sol sur lequel elles reposent. Les températures les plus basses ont été enregistrées dans les ruches recouvertes d'un badigeon à la chaux $\left(37^{\circ} 8 \mathrm{C}\right)$ ou protégées par l'ombre $\left(32,3^{\circ} \mathrm{C}\right)$. Les ruches recouvertes d'une peinture à l'aluminium ou d'une peinture bleue s'échauffent davantage (respectivement $50,0^{\circ} \mathrm{C}$ et $48,8^{\circ} \mathrm{C}$ ). Les ruches posées sur une pelouse verte s'échauffent moins.

\section{MATÉRIEL ET MÉTHODES}

Dans les expériences décrites ci-après, nous avons utilisé uniformément des ruches de type Langstroth-Root peuplées d'abeilles de race italienne (Apis mellifica var. ligusitica).

Les mesures de températures ont été effectuées à l'intérieur des ruches au moyen de thermocouples cuivre-constantan de $\mathrm{r} 80 \mathrm{~mm}$ de longueur, reliés à un potentiomètre "Skin temperature meter " de la Cambridge Instruments. Tous les essais ont été effectués en Israël, soit à Rehovot. soit à Yad-Mordecaï.

\section{RÉSULTATS}

\section{A. EFFICACITÉ DE LA RÉGULATION THERMIQUE CHEZ LES ABEIILES}

DANS IES CONDITIONS NATURELLES

Les expériences dont l'exposé va suivre ont été réalisées en octobre 1958 . Elles nous ont permis d'étudier la régulation thermique des colonies d'abeilles dans différentes conditions de température et le comportement des ouvrières assurant cette régulation.

Les mesures de température ont été faites dans des ruches de population semblable couvrant 20 cadres, mais placées dans des conditions différentes du point de vue de la protection contre l'échauffement. Une ruche était enduite d'une peinture à l'aluminium, sauf le toit qui est resté en zinc brut. Une seconde ruche était badigeonnée entièrement au moyen de lait de chaux. Une troisième était protégée 
par un abri artificiel constitué par un parasol soutenu par quatre piquets. Cet abri ne fournissait de l'ombre que pendant la période où le soleil est très haut; le matin et l'après-midi les parois de la ruche recevaient les rayons obliques.

La température au soleil et à l'ombre ainsi que la vitesse du vent ont été enregistrées au rucher pendant toute la durée des expériences (fig. I). La température

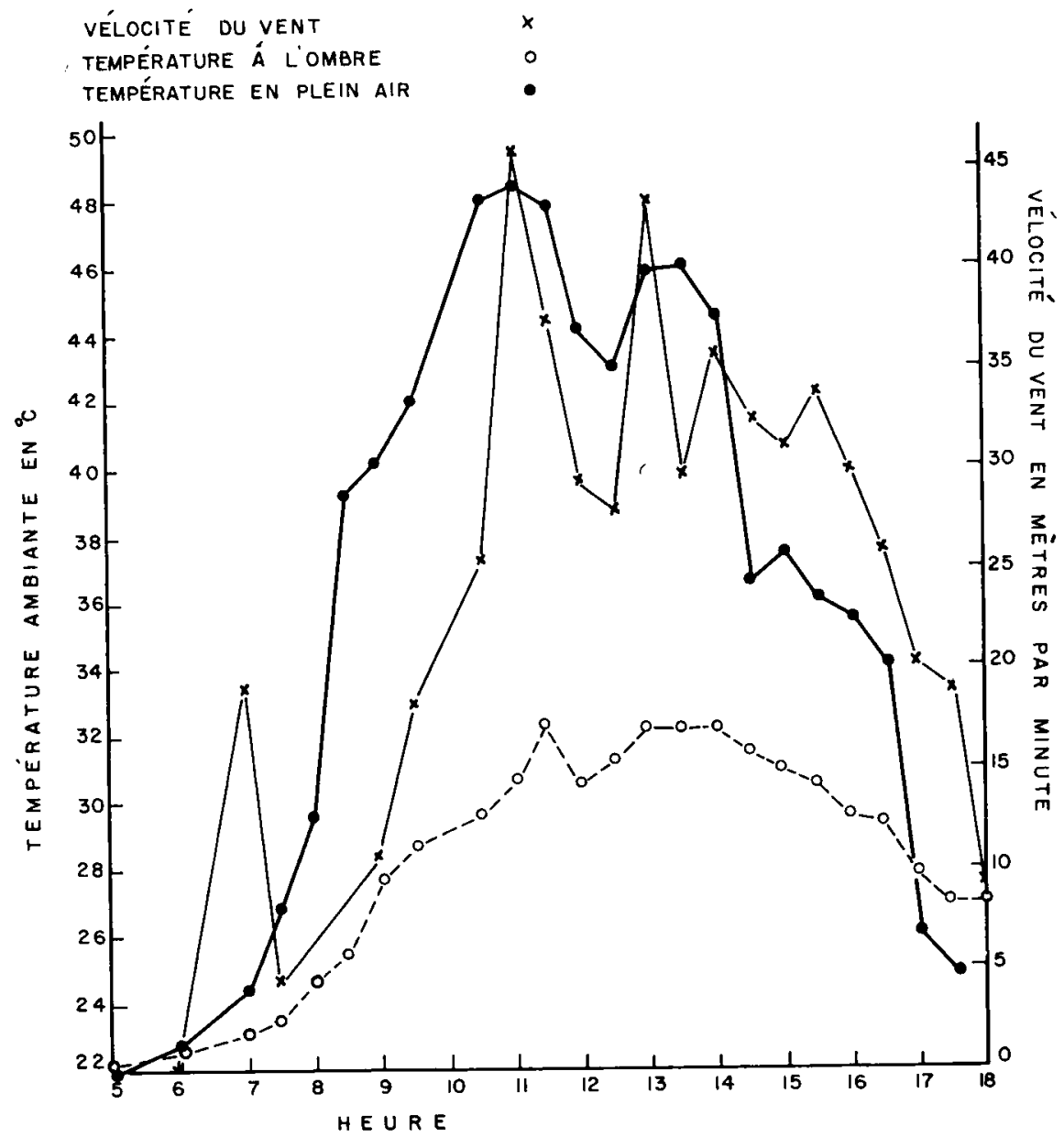

FIG. I. - Fluctuations journalières de la température et de la vélocité du vent (Rehovot, 24. X. 1958)

au soleil a été obtenue par un thermocouple placé dans un globe noirci (LENSKY, I963).

Les température à l'intérieur des ruches a été établie au moyen de thermocouples installés aux points suivants (fig. 2) :

I) Au milieu d'un rayon de miel, dans l'espace compris entre les rayons 9 et ro, du côté de la paroi nord.

2) En haut d'un rayon, dans l'espace compris entre les rayons 8 et 9 . 
3) Au centre du nid à couvain, dans l'espace compris entre les rayons 7 et 8 et à l'intérieur d'une cellule contenant une larve âgée de 3 jours.

4) Au centre du passage entre le rayon I et la paroi sud.

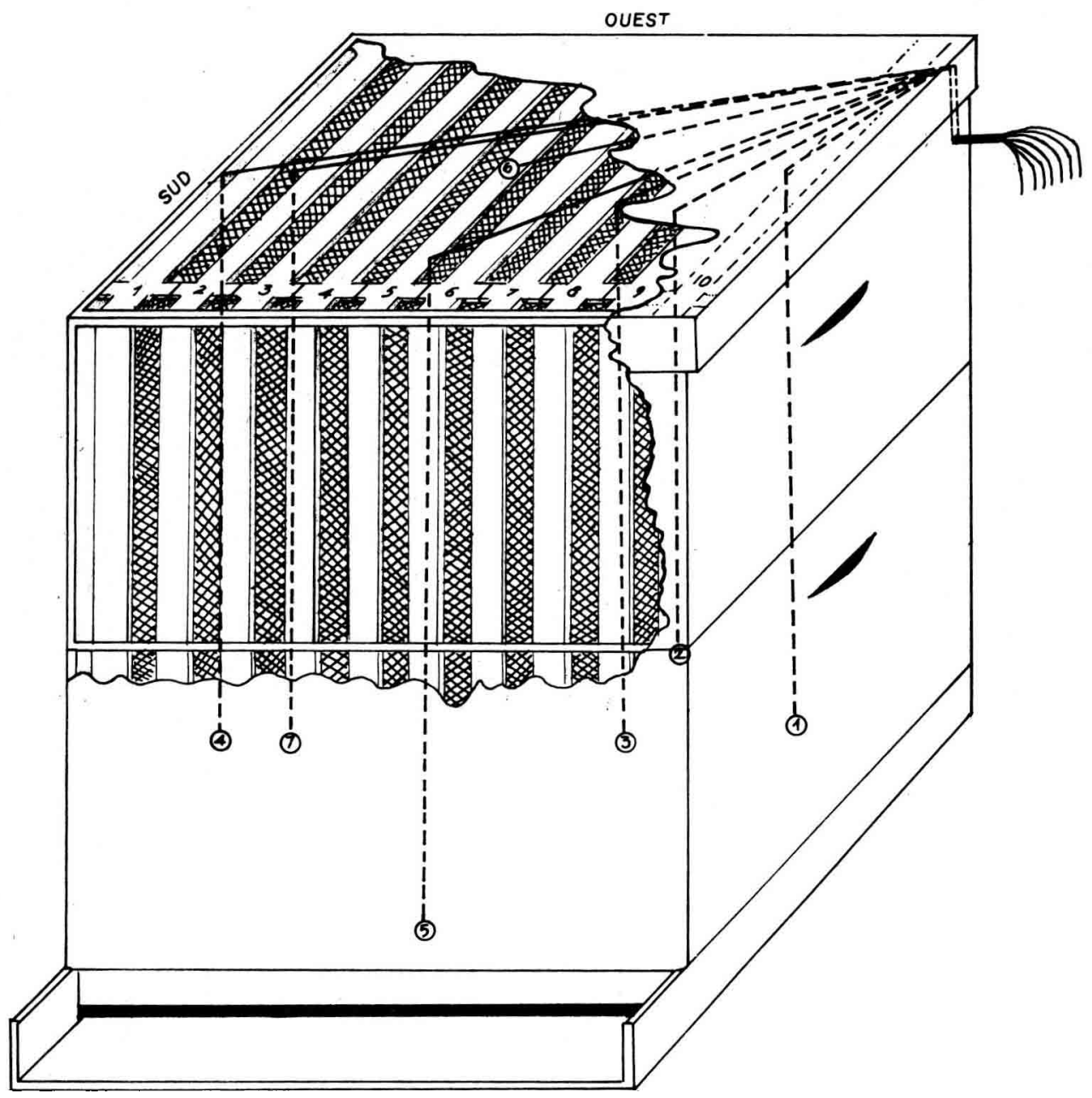

FIG. 2. - Les différenls points de mesure de la température à l'intérieur d'une nuche. Les chiffres entourés d'un cercle représentent les thermocouples, les chiffres sans cercle représentent les cadres

5) Au-dessous du rayon 5 , à $3 \mathrm{~cm}$ au-dessus du plancher et à $5 \mathrm{~cm}$ du trou de vol.

6) Dans la hausse, entre le haut du cadre 5 et la planchette couvre-cadre.

7) Dans une alvéole à miel du rayon 2, à côté de la paroi sud de la ruche.

Les variations thermiques moyennes en ces sept points de mesure et dans les trois ruches sont représentées graphiquement sur la figure $n^{0} 3$. On constate que les variations sont du même ordre dans la ruche abritée et dans la ruche blanchie à 
RUCHE PEINTE Á L'ALUMINIUM $\triangle$

RUCHE PEINTE EN BLANC $x$

RUCHE Aं L'OMBRE

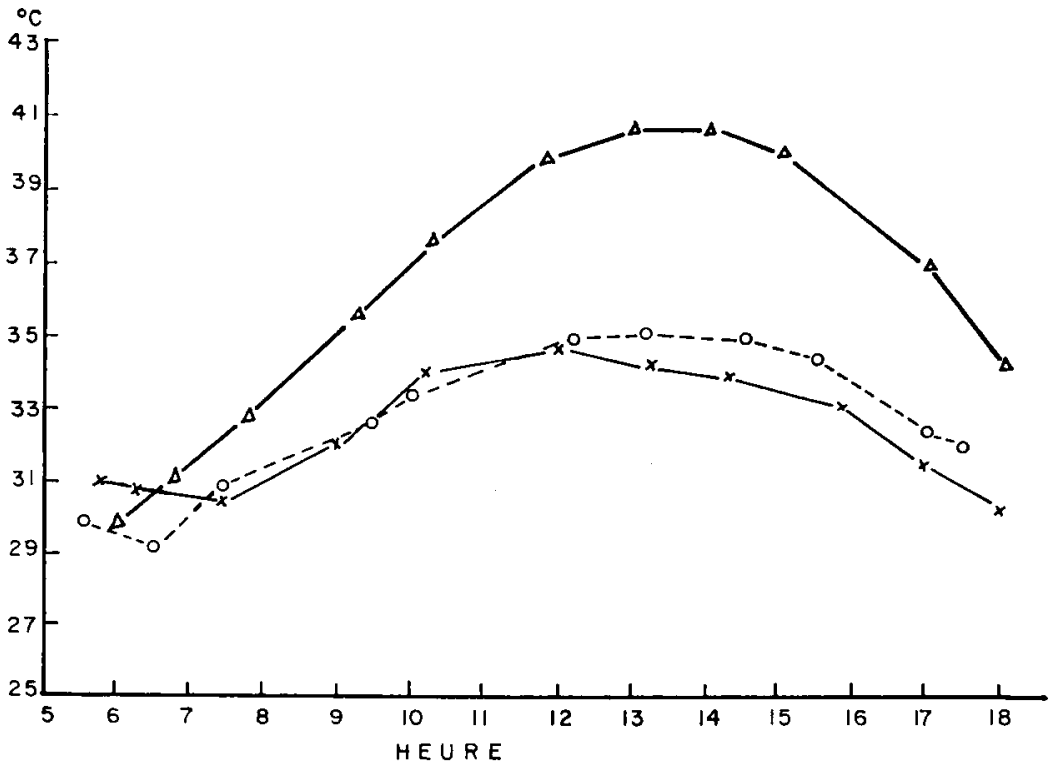

FIG. 3. - Conditions thermiques au cours de la journée (moyenne de sept différenis points de mesure) dans les ruches ayant subi irois trailements

$\begin{array}{ll}\text { RUCHE PEINTE A L'ALUMINIUM } & \Delta \\ \text { RUCHE PEINTE EN BLANC } & \times \\ \text { RUCHE A L'OMBRE } & 0\end{array}$

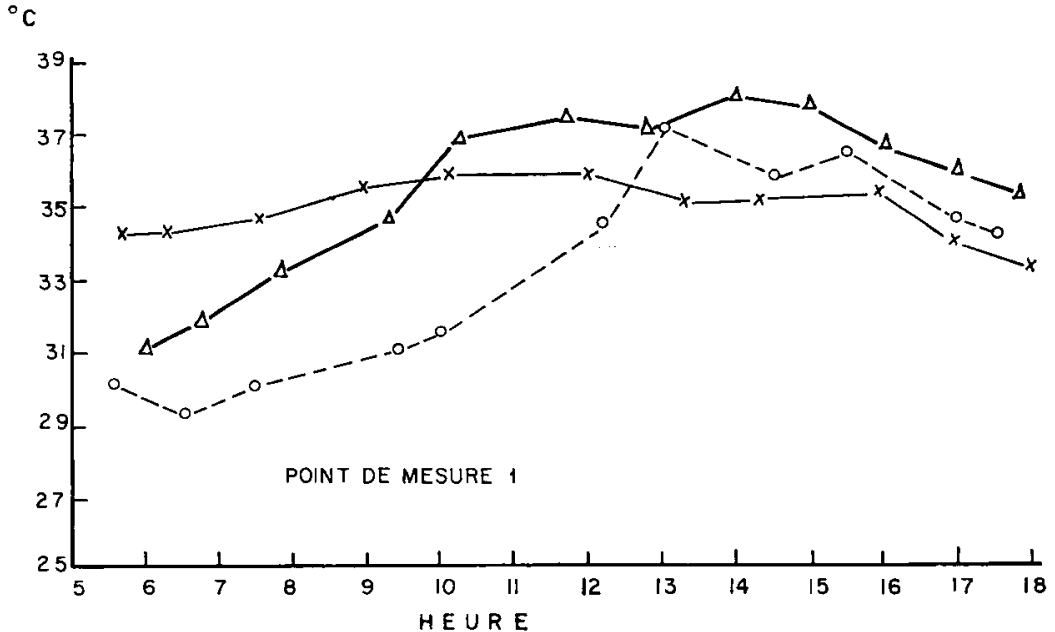

FIG. 4. - Conditions thermiques au cours de la jonmée du point de mesure $n^{\circ} 1$ dans les ruches ayant subi trois traitements 


$\begin{array}{lc}\text { RUCHE PEINTE Aं L' ALUMINIUM } & \Delta \\ \text { RUCHE PEINTE EN BLANC } & \times \\ \text { RUCHE A L'OMBRE } & \circ\end{array}$

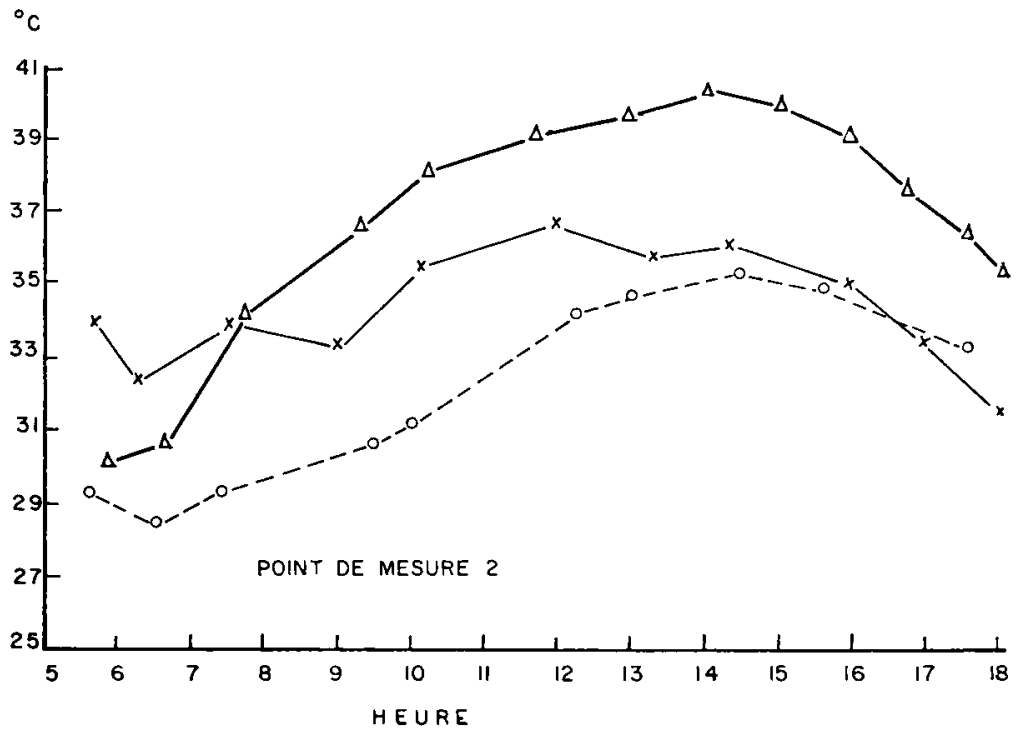

FIG. 5. - Conditions thermiques au cours de la journée du point de mesure $n^{\circ} 2$ dans les ruches ayant subi trois traitements

$\begin{array}{ll}\text { RUCHE PEINTE A L'ALUMINIUM } & \boldsymbol{\Delta} \\ \text { RUCHE PEINTE EN BLANC } & \boldsymbol{X} \\ \text { RUCHE A L'OMBRE } & \circ\end{array}$

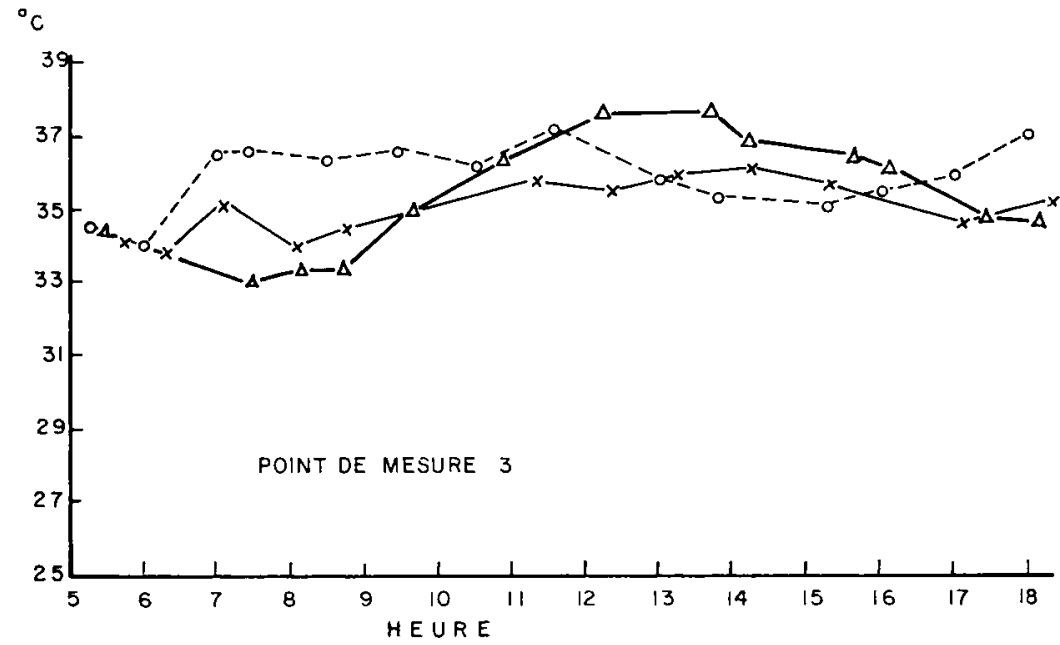

FIG. 6. - Conditions thermiques au cours de la journée du point de mesure $n^{\circ} 3$ dans les ruches ayant subi trois traitements 
la chaux. Par contre, elles sont plus élevées dans la ruche peinte à la peinture à l'aluminium.

Voyons maintenant comment se sont faites les variations de température aux différents points de mesure et en fonction du mode de protection.
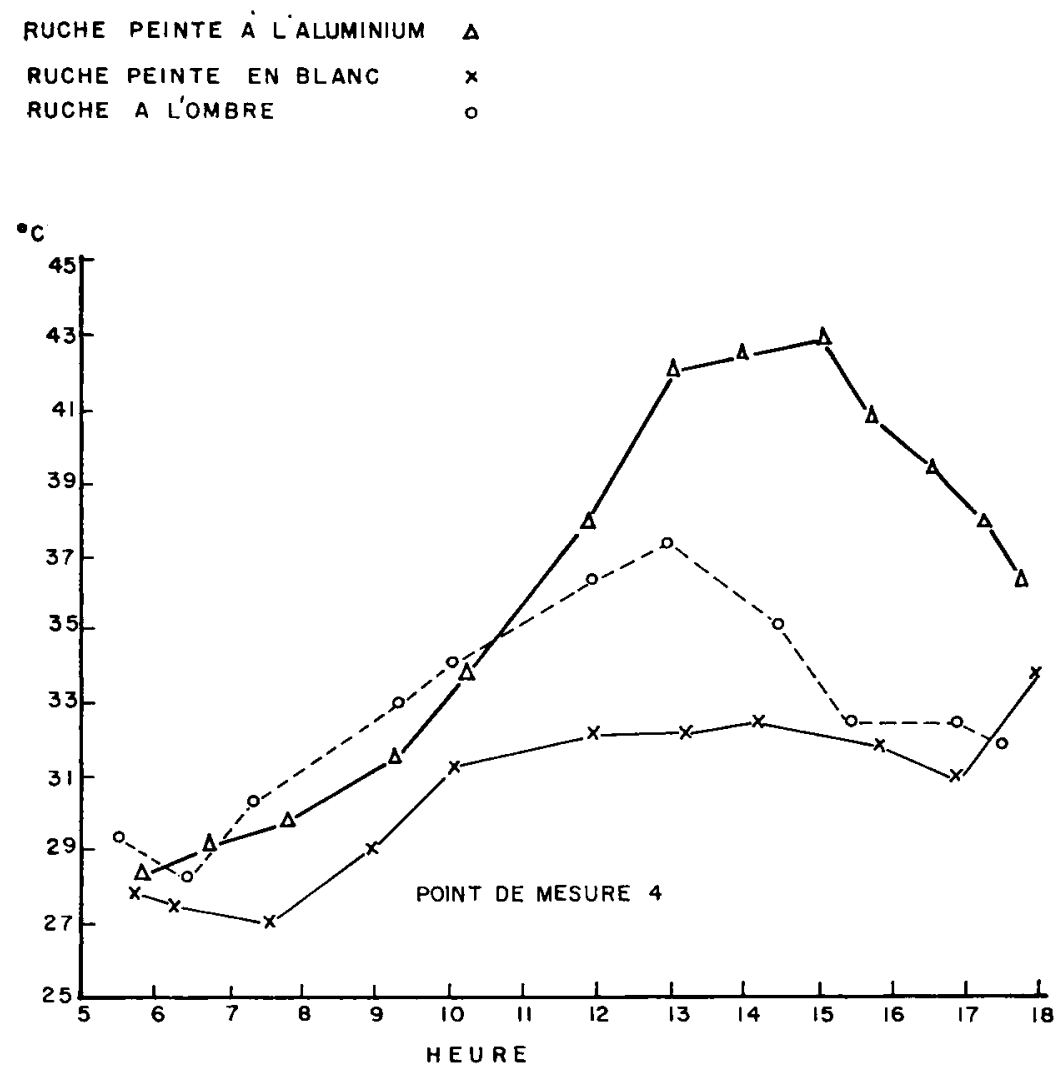

FIG. 7. - Conditions thermiques au cours de la journée du point de mesure $n^{\circ} 1$ dans les ruches ayant subi trois traitements

Point $n^{\circ} 1$ (fig. 4). Le maximum de température enregistré est de $37,9^{\circ} \mathrm{C}$ dans la ruche peinte à $1^{\prime}$ aluminium contre $35,6^{\circ} \mathrm{C}$ seulement dans la ruche peinte à la chaux.

Point $n^{\circ} 2$ (fig. 5). En ce point les variations de température sont très importantes. Rappelons que la température est prise en dehors du couvain.

Point $n^{\circ} 3$ (fig. 6). On remarque la plus grande stabilité des températures, en rapport avec la présence de couvain. 
Point $n^{\circ} 4$ (fig. 7). On note des différences remarquables en fonction de la nature de la protection. La ruche peinte à l'aluminium atteint un maximum de $42^{\circ} \mathrm{C}$ contre $33,3^{\circ} \mathrm{C}$ pour la ruche blanchie à la chaux.

Point no 5 (fig. 8). La température la plus élevée a été mesurée dans la ruche peinte à l'aluminium $\left(43,7^{\circ} \mathrm{C}\right)$ et la plus basse dans la ruche à l'ombre
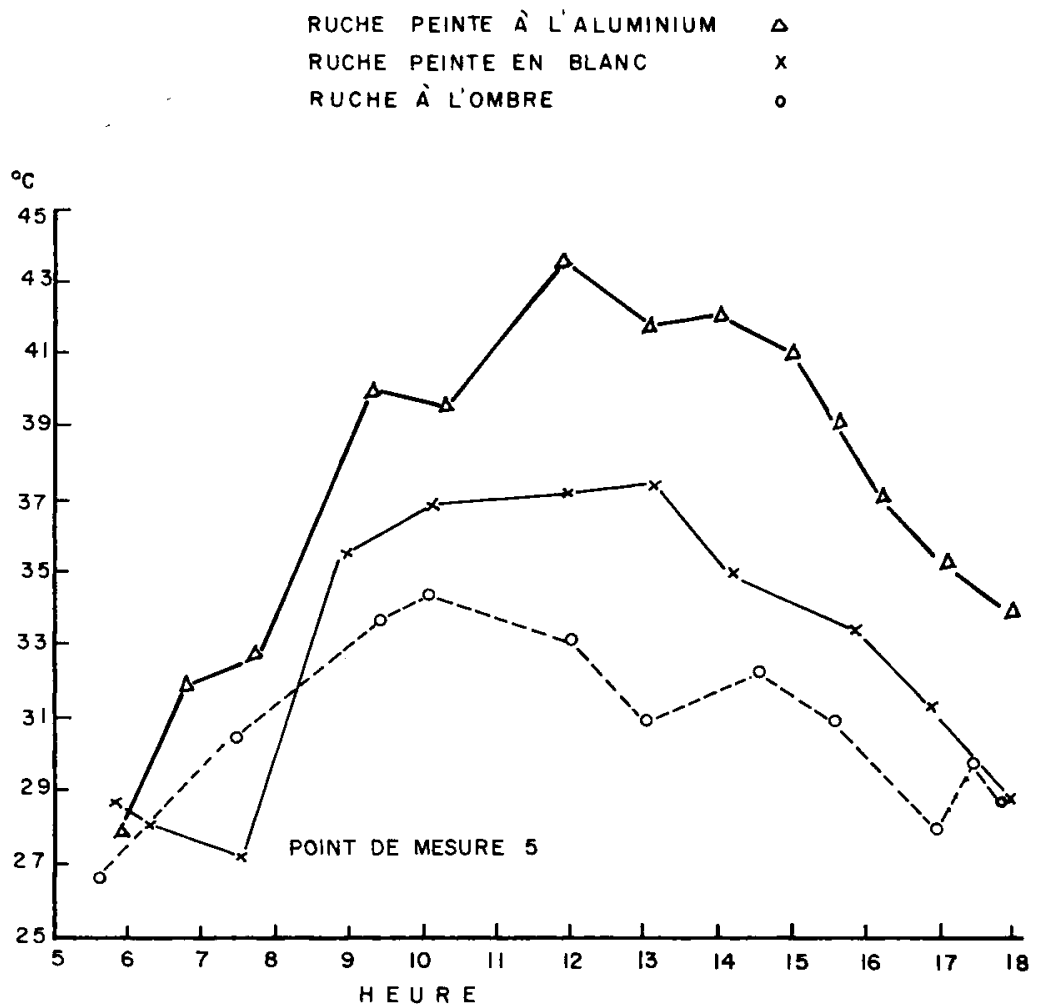

FIG. 8. - Conditions thermiques au cours de la journée du point de mesure $n^{\circ} 5$ dans les ruches ayant subi trois traitements

Point $n^{\circ} 6$ (fig. 9). Dans la ruche peinte à l'aluminium la température a dépassé de $20^{\circ}$ celle des autres ruches. Cette différence considérable est due à la radiation solaire atteignant directement le toit, lequel n'a reçu aucune protection.

Point $n^{0} 7$ (fig. Io). La sonde se trouvait dans une cellule à miel et exposée au sud. Dans la ruche à l'ombre, l'influence des rayons obliques est évidente mais c'est encore la ruche peinte à l'aluminium qui présente le plus fort maximum. 


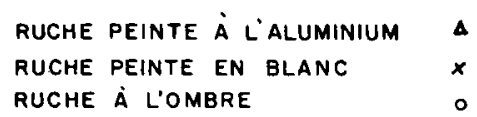

${ }^{\circ} \mathrm{C}$

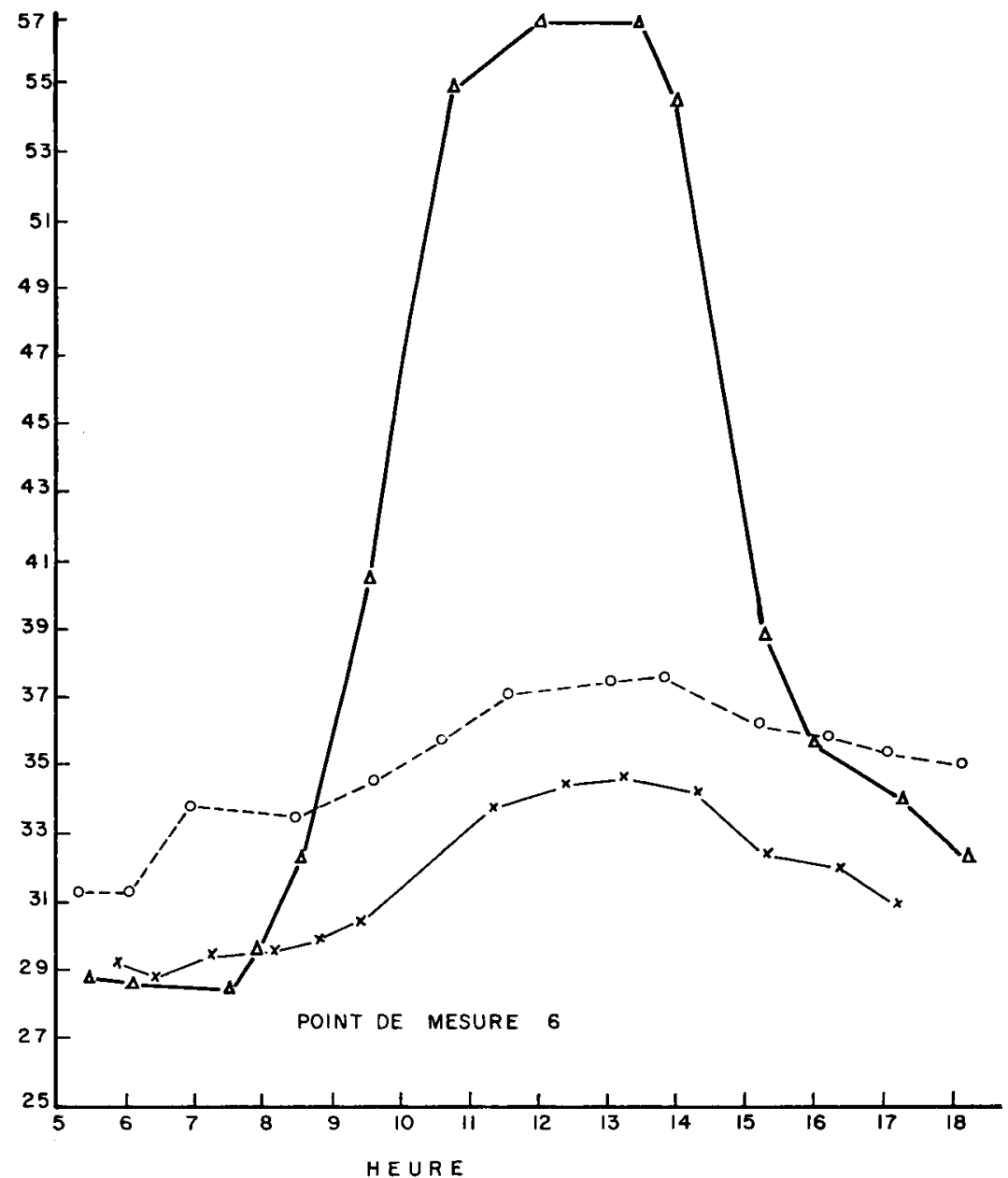

FIG. 9. - Conditions thermiques au cours de la journée du point de mesure $n^{0} 6$ dans les ruches ayant subi trois traitements 
L'activité thermorégulatrice des abeilles a été examinée parallèlement aux variations de la température à l'intérieur des ruches. Nous avons observé d'une part l'activité des ventileuses et d'autre part celle des collectrices d'eau.
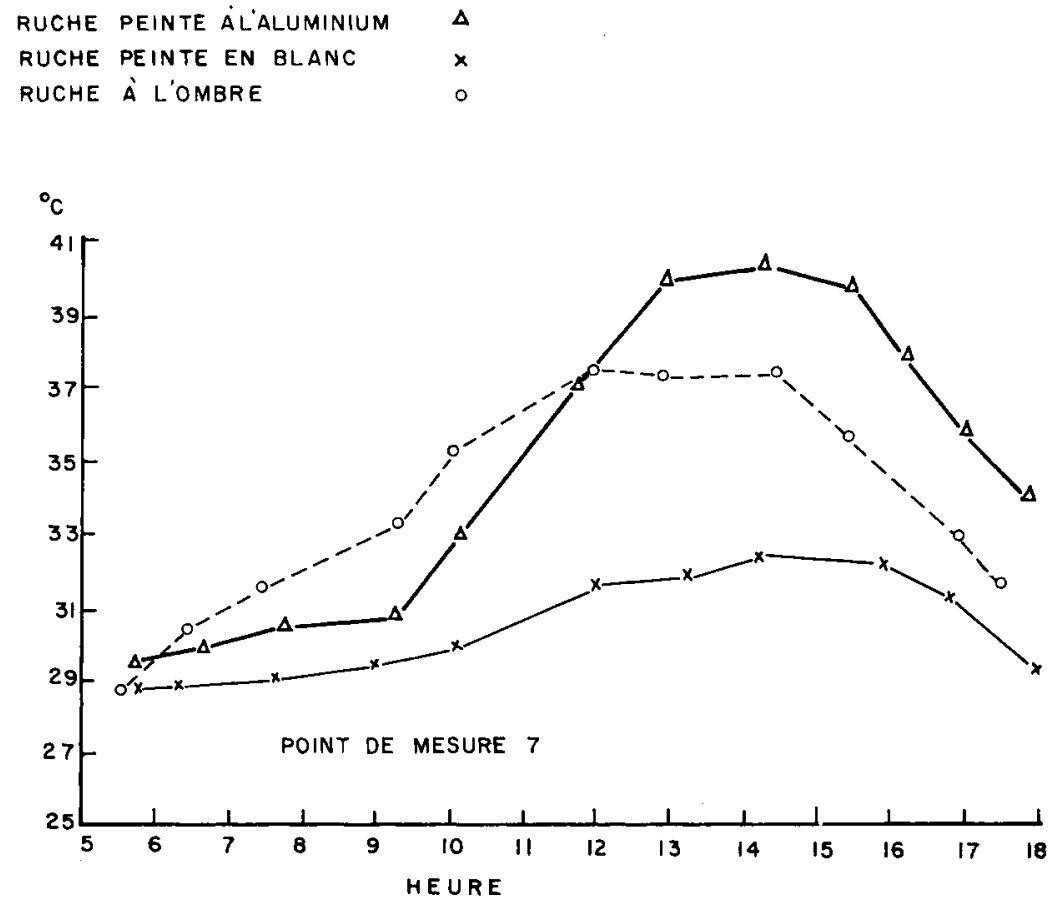

FIG. Io. - Conditions thermiques au cours de la journée du point de mesure $n^{\circ} \gamma$ dans les ruches ayant subi trois traitements

\section{Activité des ventileuses.}

L'activité des ventileuses a été déterminée heure par heure. La qualité de ventileuse a été établie en se basant sur la définition d'HAZEIHOFF, I954. La courbe de l'activité journalière des ventileuses est représentée à la figure II. Les valeurs sont exprimées en " moving average " (ARKIN et CoL'TON, I955) et calculées d'après les données absolues.

L,es tendances de l'activité des ventileuses sont semblables dans les trois ruches mais leur niveau diffère selon la protection. La ruche peinte à l'aluminium se distingue par une très forte activité. Dans la ruche à l'ombre, une montée de l'activité est enregistrée jusqu'à $I 2 h$; il y a ensuite un palier jusqu'à $I_{5} h$. Entre $I_{2}$ et $I_{5} h$, la protection offerte par l'abri est à son maximum. Dans la ruche peinte à la chaux, le niveau d'activité est le plus bas.

Activité des collectrices d'eau.

L'activité journalière des collectrices d'eau a été établie par examen du jabot d'échantillons de 50 abeilles butineuses capturées au trou de vol au moment de leur 
atterrissage au moyen d'un aspirateur. Les abeilles captives, transportées aussitôt au laboratoire ont été légèrement pressées entre deux objets de façon à obtenir la régurgitation d'une gouttelette de liquide (PARK, I932). Celui-ci étant déposé entre les prismes d'un réfractomètre $\mathrm{ABB}$ É, nous en déterminions immédiatement la teneur en sucres. Nous avons considéré comme collectrice d'eau toute abeille dont le jabot contenait un liquide renfermant moins de io \% de sucre. Les autres étaient considérées comme butineuses de nectar.
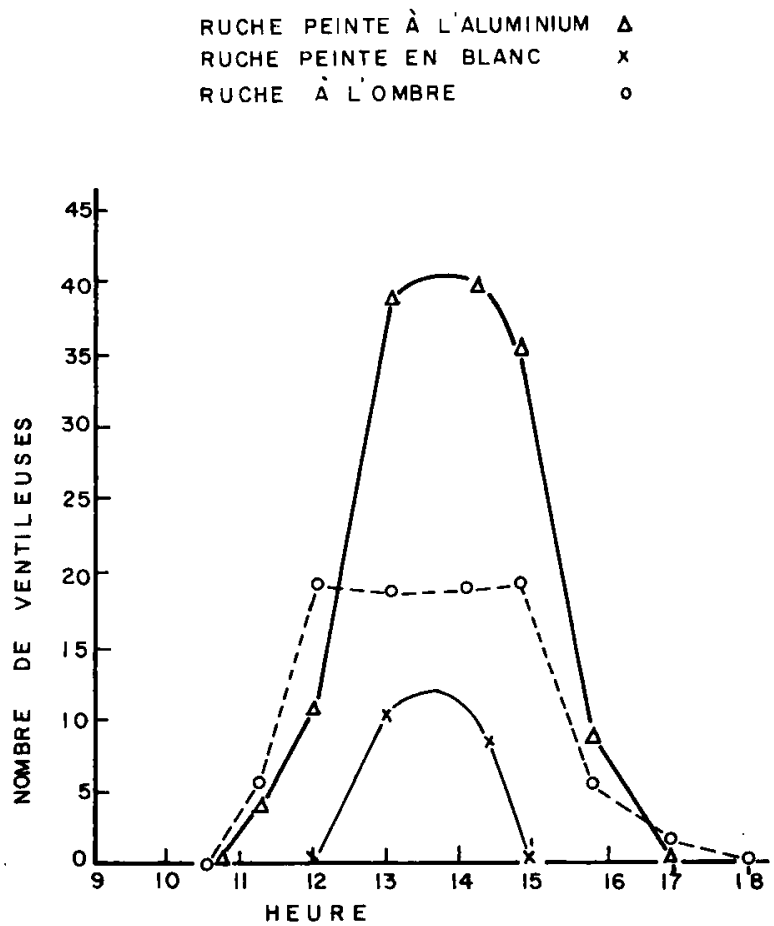

FIG. II. - - Adtirité journalière des ventileuses dans les ruches ayant subi les trois traitements

L'activité des collectrices d'eau est représentée sur la figure I2 en p. Ioo du nombre d'abeilles de l'échantillon prélevé. On constate que l'activité a été beaucoup plus grande dans la ruche peinte à l'aluminium et pendant les heures les plus chaudes que dans les autres ruches. L'apport d'eau dans la ruche badigeonnée à la chaux a commencé tôt le matin, a progressé pendant le midi et s'est poursuivi jusqu'au soir. Il convient de noter que cette colonie possédait plus de couvain ouvert que les deux autres. L'activité des collectrices d'eau dans la matinée est sans doute en rapport avec les besoins du couvain jeune. Pendant les heures chaudes l'eau nécessaire à la régulation thermique accroît les besoins. Dans la ruche abritée, on note une chute de l'approvisionnement en eau qui correspond bien à la période de protection maximum contre le rayonnement. 
Si, au lieu de ne tenir compte que des collectrices d'eau, on établit des courbes exprimant le pourcentage des abeilles dont le jabot est chargé de liquide, qu'il s'agisse d'eau ou de nectar, on obtient le graphique représenté à la figure I3. Les
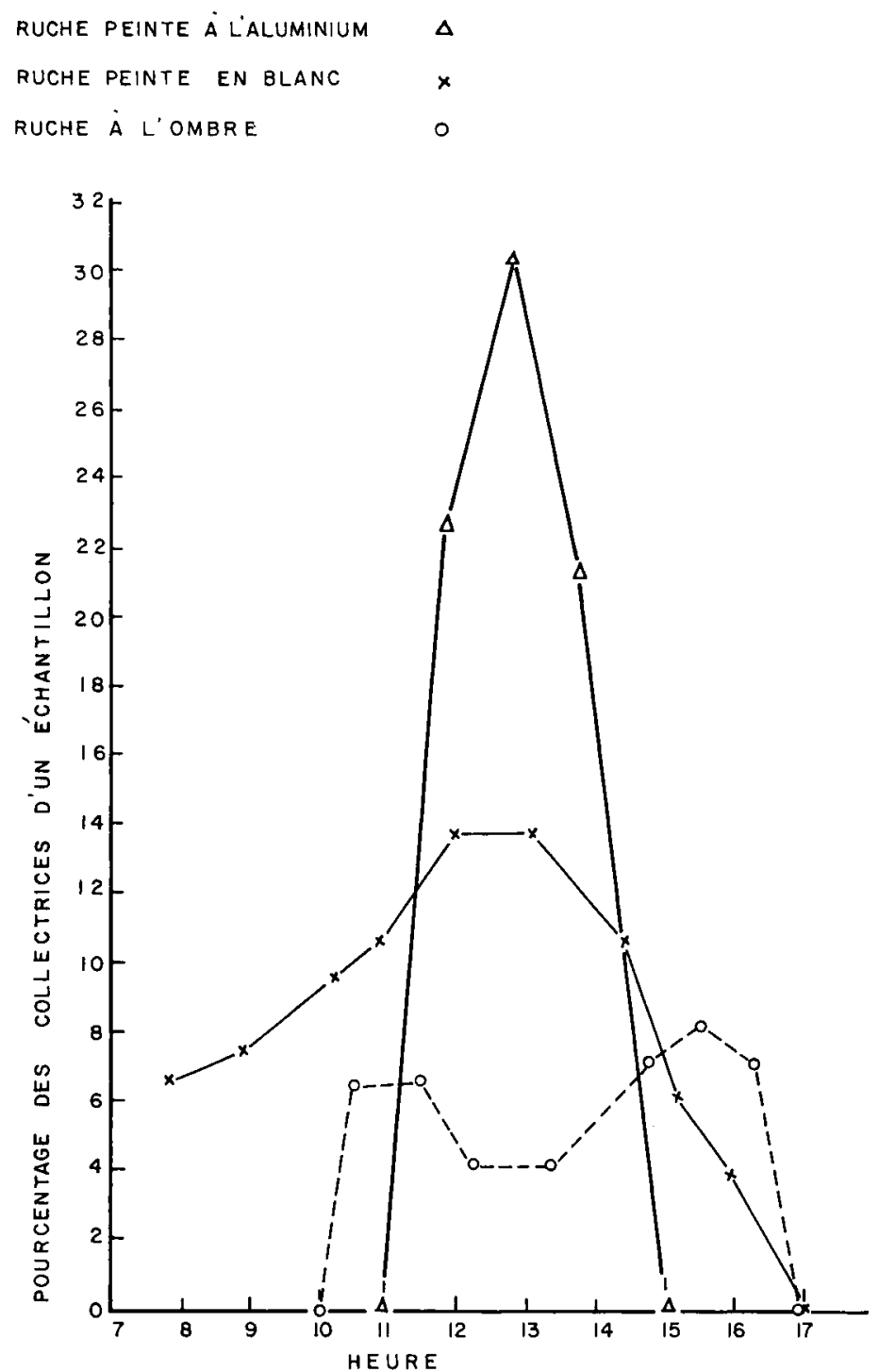

FIG. 12. - Aclivité journalière des collectrices d'eau dans les ruches ayant subi les trois traitements

figures I2 et I 3 sont donc complémentaires. On constate que pour toutes les ruches, il y a une forte activité matinale en ce qui concerne la récolte du nectar; en effet les collectrices d'eau sont peu nombreuses à ce moment. Alors que pour la ruche peinte à la chaux et pour la ruche à l'ombre, le pourcentage des abeilles dont le jabot 
contient du liquide ne cesse de baisser pendant la période la plus chaude. il remonte pour la ruche peinte à l'aluminium. Ce phénomène traduit bien les apports d'eau importants mis en évidence sur la figure I 2 et qui correspondent à une intense activité thermorégulatrice.

$\begin{array}{ll}\text { RUCHE PEINTE Aं 'ALUMINIUM } & \Delta \\ \text { RUCHE PEINTE EN BLANC } & \times \\ \text { RUCHE Aं L'OMBRE } & 0\end{array}$

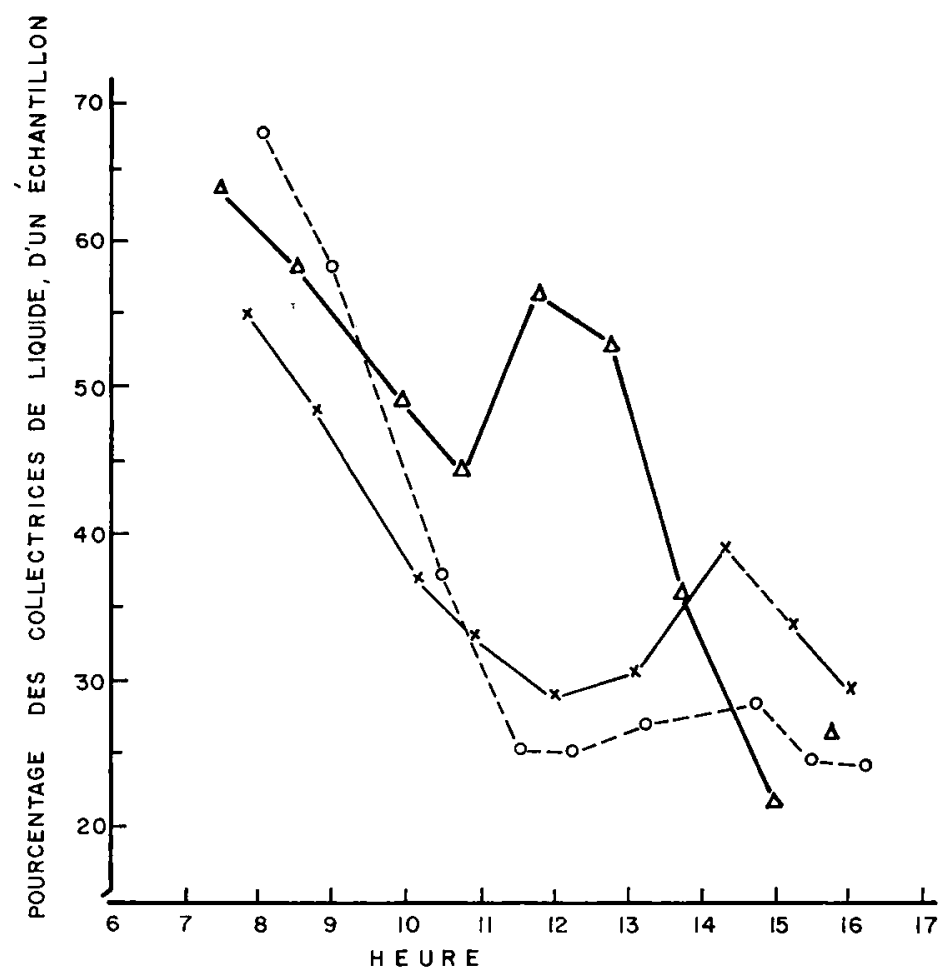

FIG. I3. - Fluctuations journalières du pourcentage des collectrices du liquide

Relation entre la température du nid à couvain et l'activité thermorégulatrice des abeilles.

Cette relation est la plus significative dans la ruche peinte à l'aluminium qui a été soumise à des températures plus fortes que les deux autres ruches. Si nous partons sur un même graphique (fig. I4), la température du nid à couvain (point de mesure $n^{\circ} 3$ ), le nombre des ventileuses et le nombre des collectrices d'eau enregistrés dans la ruche peinte à l'aluminium, nous constatons qu'il existe une corrélation très nette entre les trois phénomènes.

Activité des butineuses aux sources d'eau et de nectar.

Afin de contrôler par des observations à l'extérieur celles que nous faisons dans les ruches ou à leur entrée, nous avons procédé à des dénombrements d'abeilles 
sur une source de nectar importante, les fleurs de Salvia leucophylla, et au bord d'un bassin d'eau douce. Dans ce dernier cas, nous comptions les ouvrières sur une longueur de I mètre de long du bassin en question.

La figure I5 représente l'activité des butineuses sur les deux sources exprimée en pourcentage de l'activité totale pour la journée. On remarque que les visites aux fleurs de Salvia ont lieu surtout le matin et que le maximum est atteint à

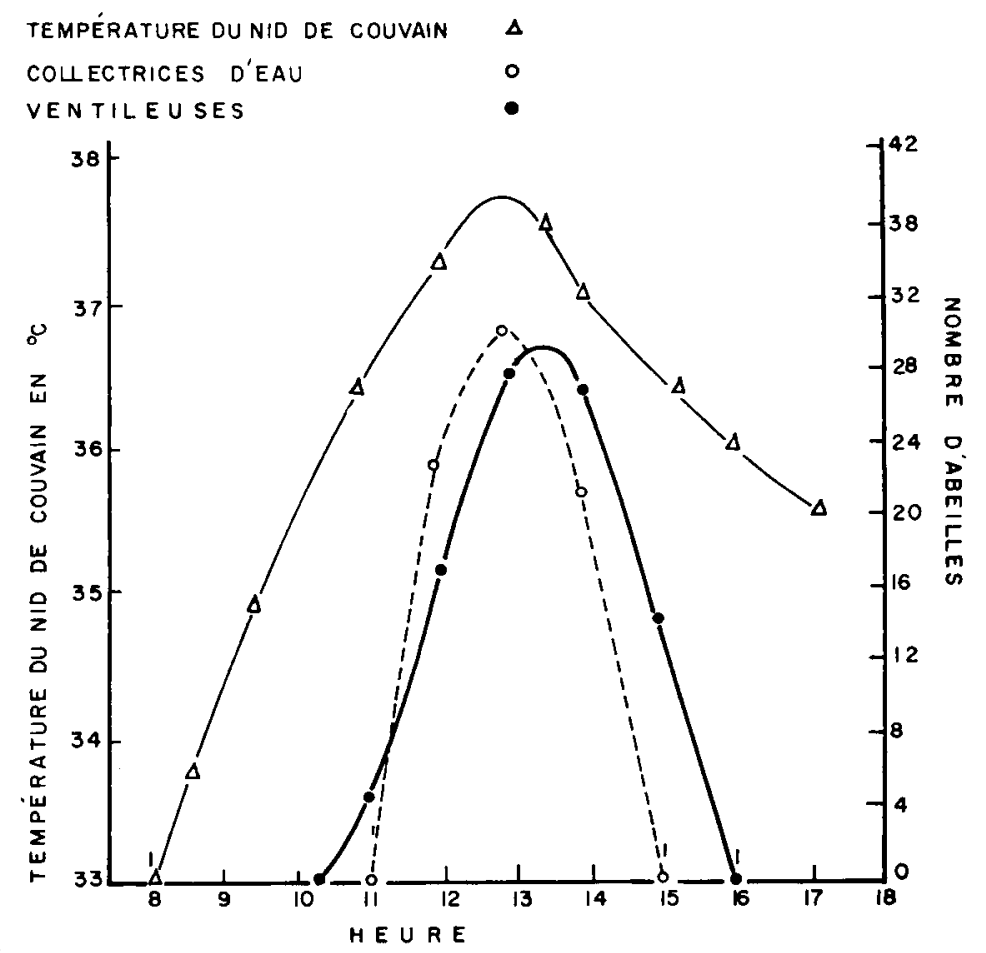

FIG. 14. - Relation entre la température du nid de convain et l'activité journalière des ventileuses et des collectrices d'eau dans la ruche peinle à lahminium

II heures. La récolte de l'eau est nettement décalée par rapport à celle du nectar et elle n'atteint son maximum qu'à $I_{4}$ heures. Ce pic correspond au maximum de l'activité thermorégulatrice des abeilles tel qu'il est mis en évidence sur les figures I I et I2. Bien entendu, les observations faites à l'extérieur constituent une moyenne de toutes les ruches en expérience placées dans des conditions thermiques fort différentes.

\section{B. - INFluence D'Un appport D'Eau dans les RUChes SUR LA PONTE DE LA REINE}

Sur les conseils du pr R. Chauvin, nous avons effectué un certain nombre d'expériences sur l'influence possible d'un apport artificiel d'eau dans la ruche sur la ponte de la reine. Les essais ont été réalisés au cours du printemps et de l'été $\mathrm{I} 96 \mathrm{r}$ sur Io ruches Langstroth-Rooth peintes en blanc. Toutes les colonies avaient des reines 


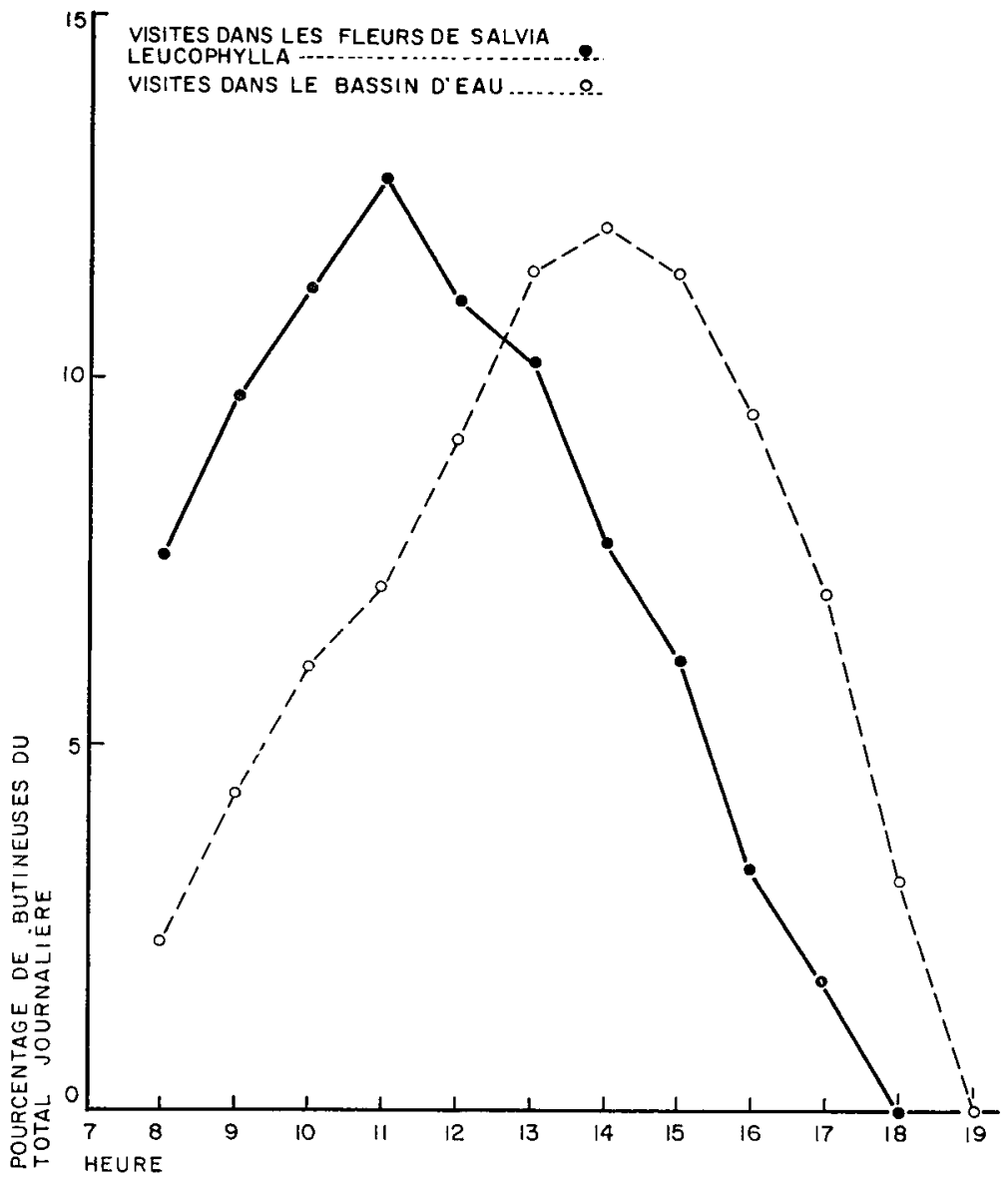

FIG. I5. - Fréquence de visites des butineuses aux sources de nectar et d'eau (Rehovot, 24. X. 1958)

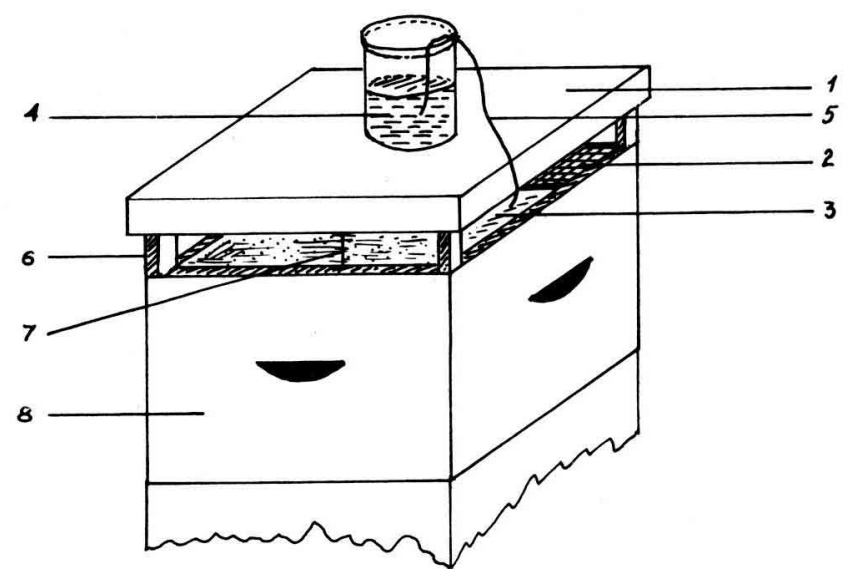

FlG. т6. - Dispositif pour l'aspersion d'eau dans une ruche

1. Toit de la ruche ; 2 . Toile de l'aération ; 3. Chiffon de laine ; 4. Récipient d'eau ; 5. Tuyau d'eau ; 6. Sup. port de toit; 7 . Espace de $5 \mathrm{~cm}$ entre le toit et la toile; 8 . Hausse de la ruche. 


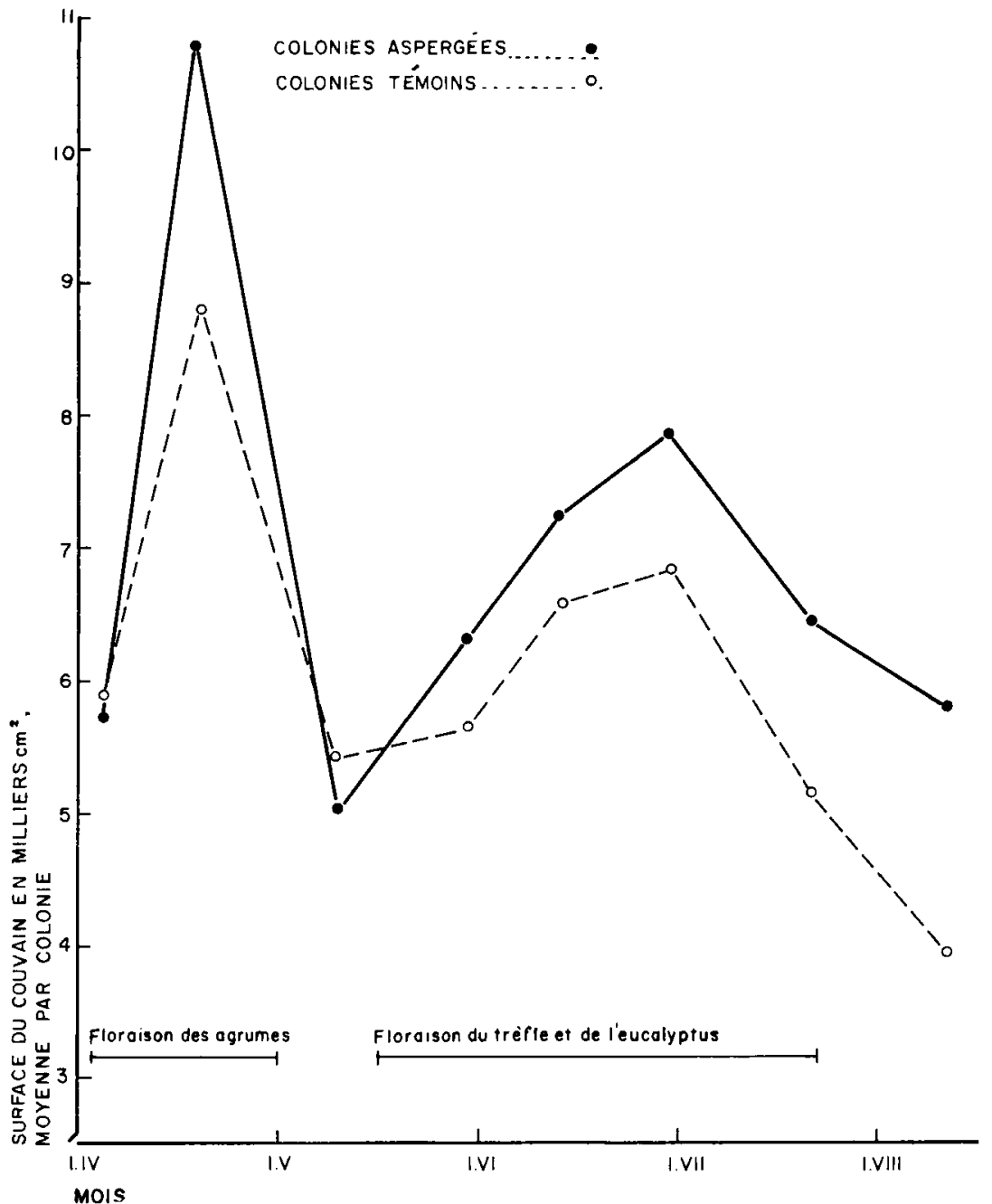

FIG. I7. - Fluctuations des pontes dans les colonies aspergées et coloniés lémoins pentant le printemps el l'élé ig6r à Rehorol et à Beit-Dagan.

\section{TABLEAU I}

Infuence sur la ponte des reines de l'apport d'eau dans les ruches : Analyse de variance

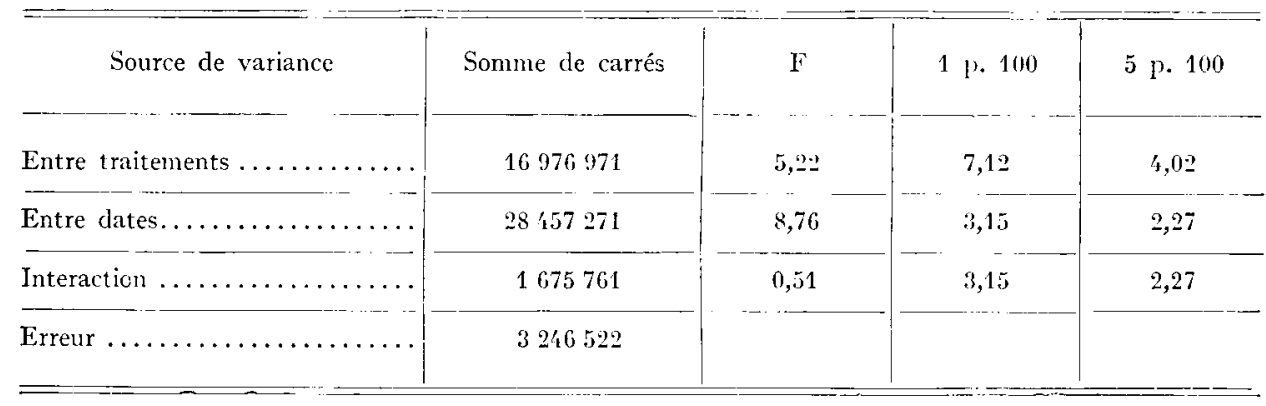


âgées d'un an. Afin d'éviter toute perturbation de la ponte des reines, nous n'avons pas utilisé de grilles à reines et nous n'avons procédé à aucune extraction de miel. L'opération a débuté à Rehovoth au printemps, au moment de la miellée des Agrumes. Ensuite, les ruches ont été transportées en mai à Beit-Dagan pour y profiter d'une miellée de Trèfle et d'Eucalyptus. Les mesures de couvain ont été faites selon la méthode décrite par FresNaye et LENSKY (I96I).

Chaque ruche mise en expérience était équipée du dispositif de distribution d'eau représenté à la figure I6. La planchette couvre-cadre est remplacée par une toile insérée dans un cadre de bois (2). Une moitié de la toile est recouverte par une étoffe de laine (3) tandis que l'autre moitié est laissée libre pour permettre les

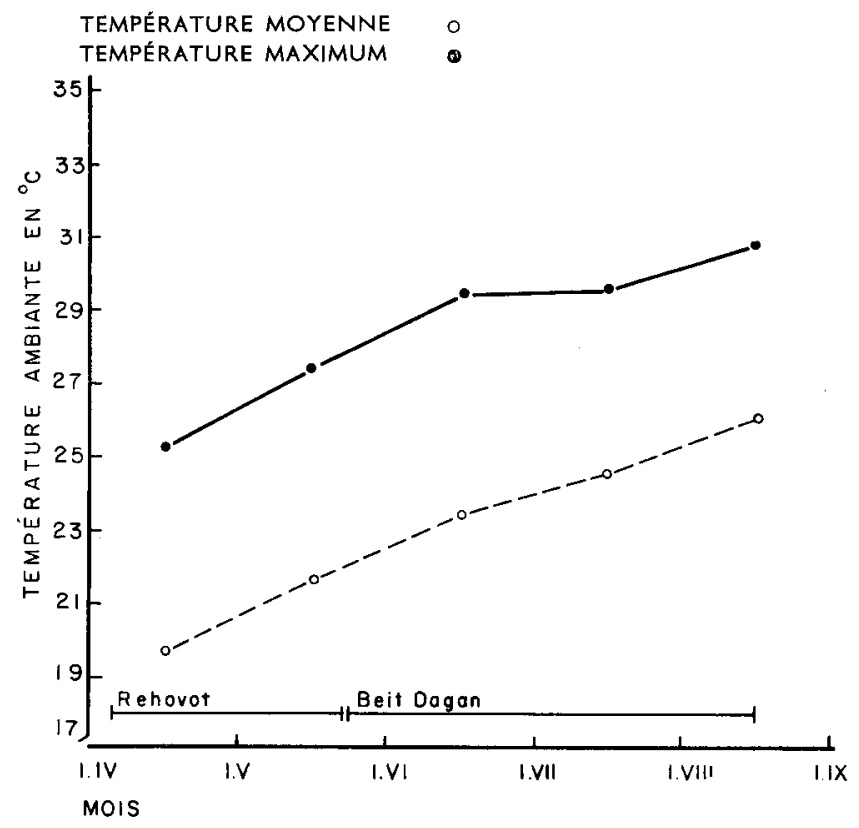

Fic. I8. - Fluctuations mensuelles de la température ambiante pendant le printemps et l'été 1961, à Rehovot et à Beit-Dagan

échanges d'air entre l'intérieur de la ruche et l'extérieur. Le toit de la ruche (I) est maintenu à $5 \mathrm{~cm}$ au-dessus du couvre-cadre au moyen de quatre petites cales. Par cet espace, on fait arriver un tube capillaire qui déverse goutte à goutte sur l'étoffe de laine l'eau d'un réservoir placé sur le toit. Le débit est de I ooo à I $300 \mathrm{~m} 1$ par 24 heures.

On a comparé l'étendue du couvain dans les ruches traitées comme il vient d'être décrit et dans les ruches témoins (fig. I7). L'allure générale des courbes est la même. La ponte présente un maximum en avril avec les apports de nectar provenant des agrumes. La fin de la miellée est marquée par une régression de la ponte. Un second maximum est enregistré en juin au moment de la floraison des Trèfles et des Eucalyptus. L'influence favorable de 1'apport artificiel d'eau aux colonies est évidente pendant toute la durée de l'expérience à l'exception du 8 mai Ig6r.

L'analyse de la variance (SNEDECoR, I959) des résultats montre que la diffé- 
rence entre ruches traitées et ruches témoins est significative au niveau de $5 \mathrm{p}$. Ioo (tabl. I). Les différences entre les dates sont significatives à I p. Ioo et l'interaction entre les traitements et les dates n'est pas significative à $5 \mathrm{p}$. Ioo.

Il ressort de ces résultats que les différences entre les surfaces de couvain des deux traitements ont augmenté graduellement avec la température ambiante (fig. r8) malgré une tendance commune aux deux groupes de colonie à réduire l'étendue de la ponte en fonction du manque de nectar.

\section{C. - INFLUENCE DU RÉGIME THERMIQUE DES RUCHES SUR LA RÉCOLTE DE MIEL}

Dans les expériences dont la description va suivre, nous avons étudié l'influence du revêtement des ruches sur leur capacité de production du miel. On sait, en effet, à la suite des expériences précédemment exposées que la nature du revêtement protecteur des ruches influe considérablement sur leur régime thermique interne et sur les nécessités de régulation par les ouvrières. Il était donc normal de rechercher si cette influence a des répercussions jusqu'au niveau de la production du miel. On a examiné également l'incidence éventuelle sur la ponte de la reine puisque les deux facteurs, taux de ponte et production du miel, sont liés ainsi que l'a montré FARRAR (I937).

L'expérience s'est déroulée au rucher de Yad-Mordecaï, à proximité d'Ascalon en I958. En février, on a choisi au hasard 15 colonies d'abeilles italiennes possédant des reines âgées de un an et toutes sœurs. Les colonies, dont les réserves de miel étaient sensiblement égales, ont été divisées en trois lots placés dans les conditions suivantes :

I) Cinq ruches peintes à l'aluminium et couvertes d'un toit non peint ont été placées en plein soleil;

2) Cinq ruches blanchies à la chaux ont été placées en plein soleil ;

3) Cinq ruches peintes à l'aluminium et couvertes d'un toit non peint ont été placées à l'ombre d'un bois d'Eucalyptus.

Les données météorologiques relatives au printemps et à l'été r958 à YadMordecaï sont consignées dans la figure I9. Nous avons considéré comme récolte de miel, le miel emmagasiné dans les hausses et d'où nous l'avons extrait. Le poids de cette récolte a été déterminé par pesée des cadres avant et après extraction du miel. I es surfaces de couvain (couvain de tous âges) ont été mesurées par la méthode de FRESNAYE et L,ENSKY (Ig6I) c'est-à-dire en déterminant les dimensions des deux axes de l'ellipse formée par le couvain. Elles sont exprimées en $\mathrm{cm}^{2}$.

L,es récoltes moyennes de miel d'agrumes de la première extraction ( 28 avril 1958) ont été semblables dans les ruches des trois lots (tab1. 2). A cette époque, les ruches n'ont pas souffert d'un échauffement exagéré car les températures ambiantes étaient relativement basses. (fig. I9) La récolte de miel s'est faite en un temps assez court et la miellée a été abondante. On n'a pas trouvé de différences entre les surfaces de couvain des ruches des différents lots. Cependant, on a trouvé une corrélation entre les surfaces de couvain et les récoltes, mais le coefficient de corrélation est assez bas : $r=0,60 \mathrm{r}(p=0,8)$. 
Par contre, l'influence du revêtement des ruches apparaît avec évidence pour les récoltes de miel effectuées pendant les mois d'été (tabl. 2). La miellée a été longue et s'est prolongée jusqu'à l'extraction du miel. L,es récoltes les plus élevées

TABLEAU 2

Influence du régime thermique à l'intérieur des ruches sur la récolte du miel et la ponte des reines (Yad-Mordecaï, 1958).

\begin{tabular}{|c|c|c|c|c|c|c|}
\hline \multirow[b]{2}{*}{$\begin{array}{c}\text { Mesures } \\
\downarrow\end{array}$} & \multicolumn{2}{|c|}{$\begin{array}{c}\text { Ruches avec peinture } \\
\text { d'aluminium en plein } \\
\text { soleil }\end{array}$} & \multicolumn{2}{|c|}{$\begin{array}{c}\text { Ruches avec peinture } \\
\text { blanche en plein } \\
\text { soleil }\end{array}$} & \multicolumn{2}{|c|}{$\begin{array}{c}\text { Ruches avec peinture } \\
\text { d'aluminium à } \\
\text { l'ombre }\end{array}$} \\
\hline & $28-4-58$ & $24-8-58$ & $28-4-58$ & $2 ' t-8-58$ & $28-4-58$ & $21-8-58$ \\
\hline $\begin{array}{l}\text { Récolte de miel en } \mathrm{kg} \text { par } \\
\text { colonie (moyenne).......... }\end{array}$ & 12,1 & 7,6 & 12,1 & 12,5 & 12,5 & 14,2 \\
\hline $\begin{array}{l}\text { Surface de couvain en milliers } \\
\text { de } \mathrm{cm}^{2} \text { par colonie (noyenne).. }\end{array}$ & 8,0 & 2,5 & 7,7 & $3, \dot{\prime}$ & $9, \overline{3}$ & 3,8 \\
\hline
\end{tabular}

\section{TEMPÉRATURE MOYENNE} TEMPÉRATURE MAXIMUM

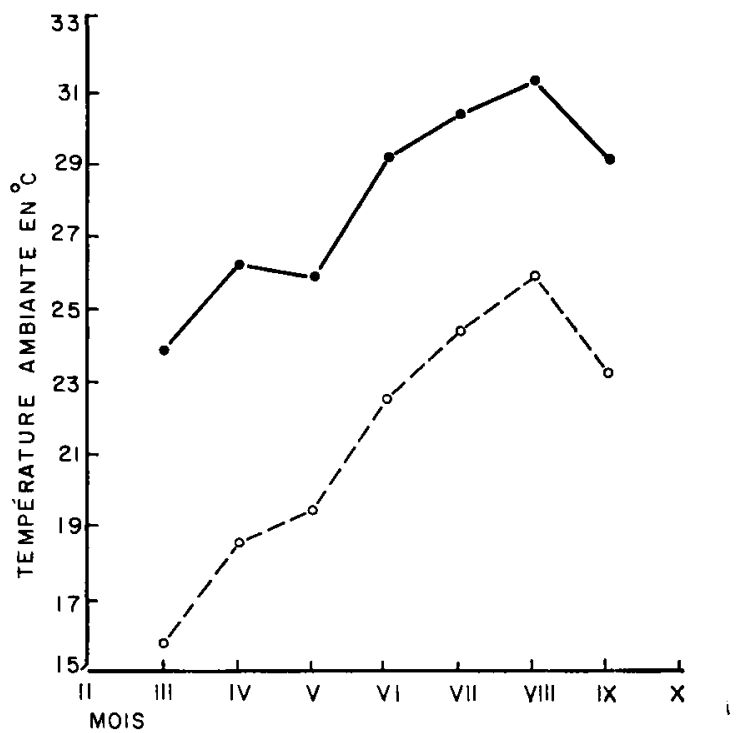

Fig. I9. - Fluctuations mensuelles de la température ambiante pendant le printemps et l'été $195^{8}$, à Yad-Mordecai.

ont été obtenues dans les ruches protégées contre la radiation solaire par l'ombre naturelle ou par un lait de chaux. Les récoltes les plus basses ont été obtenues dans les ruches peintes à l'aluminium et exposées directement au rayonnement solaire. 
La différence de récolte entre les trois lots de ruches est significative à 5 p. Ioo de probabilité.

Les surfaces de couvain des ruches protégées par l'ombre ou par la peinture à la chaux ont été supérieures à celles des ruches enduites de peinture à l'aluminium et exposées en plein soleil. Cependant, les différences ne sont pas significatives à 5 p. Ioo de probabilité.

Contrairement à ce qui se passe au printemps, nous n'avons trouvé aucune corrélation entre la surface de couvain et les récoltes du miel $(r=0,007)$.

\section{DISCUSSION ET CONCLUSIONS}

Il résulte de nos observations sur ruches vides que la meilleure protection pendant les fortes chaleurs de l'été israélien consiste d'une part en un badigeonnage à la chaux, d'autre part en tune installation sur pelouse verte et sous l'ombrage d'arbres à feuilles caduques. Ces arbres ont pour avantage de ne fournir de l'ombre que pendant la période où elle est utile.

Le régime thermique des ruches peuplées est influencé par la nature du revêtement tout comme pour les ruches vides. Ainsi, les ruches peintes à l'aluminium ont fourni les températures internes les plus élevées que nous ayons enregistrées.

La paroi sud des ruches mérite une attention particulière en raison des températures élevées qui règnent à son voisinage par suite du rayonnement solaire continu. Il est possible de réduire la température au voisinage de cette paroi par insertion de rayons de miel. Nous avons observé que les reines pondent de préférence pendant l'été du côté nord de la ruche. Par contre, en hiver, elles préfèrent le côté sud.

Les températures élevées qui ont été enregistrées sous le couvre-cadre (point de mesure $n^{0} 6$, fig. 9) dans la ruche peinte à l'aluminium sont dues à ce traitement et aussi à l'absence complète de peinture sur le toit.

La stabilité relative des températures enrezistrées dans le nid à couvain des différentes ruches est due à la régulation thermique opérée par les abeilles. L'activité de régulation se déclenche dès que la température ambiante atteint ou dépasse $25^{\circ} \mathrm{C}$ (Lindauer, I955; Vuillaume, I957). Elle est basée sur l'apport d'eau ou de nectar dans les ruches et sur la dispersion du liquide sous forme de gouttelettes ainsi, par ailleurs, que sur la ventilation. Les courants d'air créés par les ventileuses causent une évaporation du liquide et le refroidissement du nid à couvain (PARK, I960; RibBANDS, I953; HAZELHOFF, I954; LENSKY, I963). Il est évident que l'effort des abeilles pour climatiser le nid augmente leur métabolisme, leur consommation d'hydrates de carbone et, par conséquent, de miel.

L'activité thermorégulatrice la plus intensive a été observée dans la ruche peinte à l'aluminium. Elle a été provoquée par les températures élevées qui y règnent. Elle a été efficace puisque la température du nid à couvain est restée dans des limites normales.

I)ans la ruche peinte à la chaux, on a noté une surface de couvain souvent supérieure à celle qui existait dans les deux autres ruches en expérience. Ia présence de cet abondant couvain ouvert explique la forte activité des abeilles collectrices d'eau. On sait, en effet, que la colonie utilise l'eau pour diluer la nourriture 
des larves (BUTLER, I949; LINDAUER, I955) laquelle en contient de 54 à 78 p. IOO (RHEIN, I95I). Les besoins en eau sont en rapport direct avec la surface du couvain non operculé (LiNDAUER, I955). Toutefois, l'augmentation des besoins en eau pendant les heures les plus chaudes est bien en rapport avec les nécessités de la régulation thermique.

L'ensemble de ces résultats montre l'importance d'assurer aux colonies d'abeilles un approvisionnement normal en eau, surtout par temps très chaud et lorsqu'il y a manque de nectar, toutes conditions qui sont réunies surtout l'après-midi.

L'influence d'un supplément d'eau apporté par notre méthode d'aspersion est très favorable, même pendant la floraison des agrumes en avril. On peut supposer que lorsque la température ambiante ne dépasse pas $25,2^{\circ} \mathrm{C}$ (fig. I 8 ) le supplément d'eau est utilisé dans l'alimentation. En dépit de l'opinion générale selon laquelle pendant la miellée les abeilles cessent de récolter de l'eau (I)E LAYENS, I880 ; GENDOT, I907; LINDAUER, I955) nos résultats montrent une influence favorable d'un supplément d'eau sur la ponte des reines.

En ce qui concerne la baisse temporaire de la ponte dans les ruches supplémentées en eau au début de mai, nous n'avons aucune explication à proposer. Nous pensons qu'il s'agit d'un phénomène purement accidentel.

Les résultats obtenus montrent l'importance d'un approvisionnement décent en eau, dans les ruches, même lorsque les sources sont abondantes près du rucher. Cet approvisionnement serait sans doute encore beaucoup plus efficace lorsque les sources d'eau potable sont rares et éloignées du rucher.

On peut enfin affirmer une influence directe du type de protection des ruches contre la chaleur, sur la récolte de miel, tout au moins en ce qui concerne les périodes les plus chaudes. Les différences de récoltes peuvent être attribuées à 1'incidence de l'activité thermorégulatrice des abeilles. Plus cette activité est élevée, plus la consommation de miel est forte (BEUTLER, I937) plus le métabolisme est élevé (CHADWICK, I953; KAIABUCHOV, I934) et plus le glucose sanguin s'épuise rapidement (BEUTLER, I937). Mais ce n'est pas seulement l'activité musculaire qui est en cause. On sait que che\% 1'Abeille le métabolisme augmente au-dessus de $32^{\circ} \mathrm{C}$ (FARRAR, I93I; JONGBLOED et al., I934; OLAERTS, I956 ; PARHON, I9O9 ; WOODWORTH, I936). Dans nos expériences, nous avons montré qu'il règne dans la plupart des endroits de la ruche des températures supérieures à celles qu'on enregistre dans le nid à couvain, surtout lorsqu'il s'agit de ruches peintes à l'aluminium. On peut donc supposer que le métabolisme des abeilles qui séjournent dans les parties très chaudes se trouve fortement élevé, ce qui conduit à une augmentation de la consommation de miel. En fin de compte, il en résulte une baisse de la récolte, que nous avons effectivement constatée.

$$
\text { Reçu pour publication en novembre } 1963 \text {. }
$$

\section{REMERCIEMENT}

Ce travail, qui fait partie d'une thèse, a été réalisé sous la direction de M. le Professeur Z. Avidov, chef de la Section d'entomologie à la Faculté d'Agriculture de Rehovot. Je tiens à le remercier pour son intérêt et ses conseils qui m’ont guidé dans cette étude. 
J'exprime ma gratitude à M. le Professeur R. Chauvin pour ses conseils et ses encouragements dans ce travail.

Je veux enfin remercier mes collègues de la Faculté d'Agriculture, MM. A. Berman et D. Yaron, qui ont bien voulu m'aider, chacun dans son domaine.

\section{SUMMARY}

THERMAL, CONTROL OF BEE-HIVES IN SUMMER

Temperature was measured at 7 different points in populated hives coated with aluminium paint, whitewashed or protected by shade in a subtropical climate. I)iurnal activity pattern was simultaneously recorded.

In the brood nest, temperature was almost constant, while in the other parts cons:derable temperature fluctuations were found.

Strongest activity of ventilating and water carrying bees was observed in aluminimm painted hive.

Direct watering of the combs increased oviposition. This effect was more marked during the hotter season. Shading and whitewashing increased honey yields during the hottest months.

\section{RÉFÉRENCES BIBI,IOGRAPHIQUES}

AldeE W. C. et al., 1961. Principles of animal ecology. W. B. Saunders, Philadelphia and London. p. 837 . Arkin II., Colton R., 1955. Statistical methods. Barnes and Noble, New York, p. 226.

Beutler R., 1937. Über den Blutzucker der Bienen. Zeil. vergl. Physiol., 24, 71-115.

Bodenheimer F. S., Ben-Nerya A., i937. One year studies on the biology of the honeybee in Palestine. Ann. appl. Biol., 24, 385-403.

Butler C. G., I949. The honeybee. At the Clarendon Press, Oxford, p. I39.

Butler C. G,, 1954. The world of the honeybee. Collins, London, p. 226.

Chadwick I. E., I953. In K. Roeder : Insect physiology. J. Wiley and Sons, New lork, p. iroo.

De-Layens, 388o. In A. I. Root (1948) : $A B C$ and $X Y Z$ of the bee culture. A. I. Root Co., Medina, Ohio, p. 720 .

Farrar M. D., 193 . Metabolism of the adult honey bee. Jour. econ. Ent., 24, 6r I-6r6.

FARRAR C. L., 1937. The influence of colony populations on honey production. Jour. agric. Res., 54, 945-954.

Fresnaye J., Iensky Y., ig6r. Méthodes d'appréciation des surfaces de couvain daus les colonies d'Abeilles. Ann. Abeille, 4, 369-376.

Gendot G., 1907. In C. G. Butler: The honeybee. At the Clarendon Press, Oxford, p. I39.

IIAZELHOFF E. H., 1954. Ventilation in a bee-hive during summer. Physiol. comp. et CEcol., 3, 3+3-364.

Jongbloei) J., Wiersma C. A. G., 1934. Der Stoffwechsel der Honigbiene während des Fliegens. 1 Zeit. vergl. Physiol., 28, 352-357.

Kalabuchov N. I., 1934. In R. Cinauvin: Physiologie de l'insecle. I. N. R. A., l'aris, p. 917.

LENSKy Y., 1958. Some factors affecting the temperature inside hives in hot climates. Bee Irorll, 39, 205-208.

Lensky Y., 1963. Études sur la physiologie et l'écologie de l'Abeille (Apis mellifica L. var. ligustica) en Israël. Thèse, Jérusalem, p. 93.

Lensky Y., I964. Comportement d'une colonie d'Abcilles ì des températures extrêmes. J. Ins. Physiol. $10: 1-12$.

LINDALER M., 1955. The water economy and temperature regulation of the honeybee colony. Bee World, 36, 62-72;8I-91; $105-11 \mathrm{I}$.

OLAERTS L., I956. Metabolism in bees and its relations to their activity and temperature. Proc. 16th Inlern. Beekeeping Cong. Prot. No 390.

Parthon M., 1909. I.es échanges nutritifs chez les $A$ beilles pendant les quatre saisons. Amm. Sc. Nal. Zool., 9, I-58. 
PARK O. W., 1932. Studies on the changes in nectar concentration produced by the honeybee. Res. Bull. Ia. Sta., No $15 \mathrm{I}$; 209-244.

PARK O. W., ig6o. In R. GroLT : The hive and the honeybee, Dadant and Sons, Hamilton, III, p.

Ruen W. von, 1951. Lber die Enährung der Dronenmaden. Zeit. Bienenforsch., 1,, $63-66$.

RiBbands C. R., 1953. The behaviour and social life of honeybees. B. R. A. London, p. 352.

Snedecor G. W., 1959. Statistical methods. Iowa State College Press, Ames, Iowa, p. 534.

Vullause M., I957. L'importance de l'approvisionnement en eau dans la ruche. Insectes sociaux, 4, $31-41$.

Wellington W. G., 1957. The synoptic approach to studies of insects and climates. Ann. Rev. Ent, 2. $143^{-162 .}$

Woonwortil C. E., 1936. Physical factors in respiration of the honeybee. Jour. econ. Ent., 29.1128-II32 